\title{
Statin-associated Autoimmune Myopathies: A Pathophysiologic Spectrum
}

\author{
Yufan Wu, Boleslaw Lach, John P. Provias, Mark A. Tarnopolsky, \\ Steven K. Baker
}

\begin{abstract}
Background: Statins have recently been reported to cause a rare autoimmune inflammatory and/or necrotic myopathy that begins or persists after drug cessation. Methods: We report on 26 patients seen at a neuromuscular centre between 2005 and 2011 who demonstrated muscle weakness/myalgias and creatine kinase elevations during or after statin treatment with continuation of signs and symptoms despite statin withdrawal. Results: All patients were treated with immunosuppressive therapy with good response; all improved biochemically and $86 \%$ improved clinically. Sixty-five percent of patients who attempted to taper off immunosuppressive therapy relapsed. We report on a novel finding whereby five of the seven patients who underwent multiple biopsies throughout their disease demonstrated a transformation of their histological diagnosis, with four progressing from having myofibre necrosis with minimal or no inflammation to a diagnosis of polymyositis. Conclusions: This study offers preliminary evidence that statin-associated necrotizing myopathy and statin-associated polymyositis may not be separate entities but are part of the same pathophysiological spectrum. Both entities respond well to immunosuppression.
\end{abstract}

RÉSUMÉ: Myopathies autoimmunes associées aux statines: spectre physiopathologique. Contexte: On a signalé récemment que les statines pourraient causer une myopathie inflammatoire et/ou nécrotique autoimmune rare qui commence ou persiste après l'arrêt du médicament. Méthode: Nous rapportons les observations de 26 patients examinés dans une clinique neuromusculaire entre 2005 et 2011 qui présentaient de la faiblesse musculaire/des myalgies et une élévation de la créatine-kinase pendant ou après la prise de statines et dont les signes et symptômes persistaient malgré l'arrêt de la statine. Résultats: Tous les patients ont reçu des immunosuppresseurs, avec de bons résultats. Le tableau biochimique s'est amélioré chez tous et $86 \%$ se sont améliorés au point de vue clinique. Soixante-cinq pour cent des patients qui ont tenté de diminuer le traitement immunosuppresseur présenté une rechute. Nous rapportons une nouvelle constatation, soit que chez 5 des 7 patients qui ont subi de multiples biopsies au cours de leur maladie, nous avons observé une transformation de leur diagnostic histologique soit une progression de la nécrose myofibrillaire avec peu ou pas d'inflammation à un diagnostic de polymyosite. Conclusions: Cette étude présente des données préliminaires suggérant que la myopathie nécrosante associée aux statines et la polymyosite associée aux statines ne seraient pas des entités séparées mais partageraient la même physiopathologie. Ces deux entités répondent bien au traitement immunosuppresseur.

Keywords: Statin, autoimmune, polymyositis, necrotizing, myopathy, HMGCR

doi:10.1017/cjn.2014.22

Can J Neurol Sci. 2014; 41: 638-647

\section{INTRODUCTION}

3-hydroxy-3-methylglutaryl coenzyme A reductase (HMGCR) inhibitors, generically referred to as statins, lower cholesterol levels by reducing the synthesis of L-mevalonate, a crucial intermediate in the cholesterol biosynthesis pathway. Statins have been reported to be the most effective medications for controlling elevated concentrations of low-density lipoprotein cholesterol and to significantly lower the incidence of clinical cardiovascular endpoints. ${ }^{1}$ As such, they are one of the most frequently prescribed drugs, with 21.6 million prescriptions in Canada in $2006^{2}$ and 174 million in the United States in $2005 .^{3}$

In general, statin medications are well tolerated ${ }^{4}$; however, clinical manifestations of statin-induced myotoxicity have been well described. ${ }^{5-7}$ Muscle-related symptoms include myalgia $(2 \%-22 \%),{ }^{7-10}$ muscle weakness $(\sim 3 \%),{ }^{9,11-13}$ and life-threatening rhabdomyolysis (0.4 per 10,000 person-years), ${ }^{14}$ which may occur months to years after statin initiation. These symptoms most often resolve completely upon cessation of the drug. ${ }^{15}$ In contrast to these well- described clinical symptoms, the mechanisms proposed to explain acute statin myopathy are still elusive and theories include isoprenoid depletion, ubiquinone synthesis inhibition, sarcolemmal cholesterol alteration, and calcium metabolism perturbation. ${ }^{16}$

More recently, reports have emerged of patients who developed myopathies during statin therapy that later persisted or even worsened despite statin discontinuation. Most such reports describe the development of three autoimmune myopathies: typical polymyositis (PM), ${ }^{17-20}$ typical dermatomyositis

From the Stanford University School of Medicine (YW), Stanford, California 94305 USA; Department of Pathology and Molecular Medicine (BL, JPP), McMaster University, Hamilton General Site, Hamilton, Ontario L8L 2X2, Canada; Department of Pediatrics (MAT), McMaster Children's Hospital, Hamilton, Ontario L8N 3Z5, Canada; Department of Medicine (MAT, SKB), McMaster Children's Hospital, Hamilton, Ontario L8N 3Z5, Canada.

Received February 12, 2014. Final Revisions Submitted April 28, 2014. Correspondence to: Steven K. Baker, Neuromuscular and Neurometabolic Clinic, McMaster Children's Hospital, Hamilton, Ontario L8N 3Z5, Canada.

Email: bakersk@mcmaster.ca. 
(DM), ${ }^{21-26}$ and an immune-mediated necrotizing myopathy (IMNM). ${ }^{27,28}$ These conditions will be herein referred to as statinassociated autoimmune myopathies (SAMs). Although inclusion body myositis (IBM) muscle biopsies show inflammation patterns similar to those of $\mathrm{PM},{ }^{29} \mathrm{IBM}$ is not included as a SAM because IBM patients have a distinct form of weakness and these patients do not maintain a response to immunosuppression. ${ }^{30}$ Evidence also suggests IBM may be myodegenerative. ${ }^{31}$ A previous study reported on potential mechanistic findings for one of the SAMs: statins increased expression of autoantibodies against HMGCR in patients with IMNM, and this prolongs the immune response as HMGCR becomes highly expressed in regenerating muscle fibres. ${ }^{32} \mathrm{We}$ completed a retrospective case series analysis to investigate the clinical, laboratory, and muscle biopsy findings of patients diagnosed with one of the SAMs. In addition to providing further clinical details about the SAM subgroups, we report that five of seven patients had changing histological diagnoses, namely, from IMNM to PM, with repeat biopsies. To our knowledge, this is the first study that demonstrates in multiple patients a biopsy-proven transformation with serial biopsies. Our findings suggest that PM and IMNM are part of a single pathophysiological spectrum as opposed to distinct entities. Further support for "lumping" comes from the fact that all SAM subgroups showed a good response to immunosuppression.

\section{Patients and Methods}

\section{Ethics Statement}

The study protocol was approved by the Hamilton Health Sciences/Faculty of Health Sciences Research Ethics Board. The need for informed consent from participants was waived by the institutional review board (TCPS2 Article 2.2).

\section{Data Collection}

We retrospectively reviewed the medical records of all patients diagnosed at the McMaster University Neuromuscular and Neurometabolic Clinic between 2005 and 2011 with an autoimmune myopathy (i.e., PM, DM, IMNM, and nonspecific inflammatory myopathy [NSIM]) associated with statin therapy (i.e., current or discontinued within 6 months before initial visit). After investigation, all patients who remained on a statin had it discontinued by one of the investigators at the clinic (M.A.T. or S.K.B.). Records were taken from our database of clinical, biological, and pathological diagnoses of all patients who had a muscle biopsy and are monitored at the clinic.

A comprehensive collation of chronologically ordered information was extracted on the number, type(s), dosage(s), and duration(s) of statins or other lipid-lowering drugs (LLDs) used; indicators of myopathy (i.e., myalgias, weakness, dysphagia, rhabdomyolysis, renal dysfunction, hospitalization, hyperCKemia [elevated creatine kinase $\{\mathrm{CK}\}$ levels in blood]); findings from muscle biopsy, electromyography (EMG), and quantitative muscle strength tests; instances and consequences of rechallenge of a statin or other LLD; the use of concomitant drugs with pharmacologic interaction with statins or other myotoxic drugs, with the list of drugs cited in previous reviews, ${ }^{33-35}$ conditions or behaviours that may predispose to statin-associated myopathy (i.e., mixed connective tissue disease, paraneoplastic syndromes, ${ }^{27}$ obesity, hypothyroidism, hypertension, diabetes, alcohol use ${ }^{34}$ ); serological markers of disease and inflammation (almost all cases were collected before the discovery of the anti-HMGCR antibody and serum samples were not stored for testing); and the outcomes of introducing and discontinuing immunosuppressive therapy.

\section{Inclusion Criteria}

After all available information was reviewed, only patients with probable SAMs were included. Inclusion criteria consisted of weakness, myalgia, and/or hyperCKemia (>220 IU/L in men; $>170$ IU/L in women) while on statin therapy. These clinical signs and symptoms had to persist or worsen for at least 2 months after discontinuing the statin and have indications for immunosuppressive therapy (i.e., prednisone, methylprednisolone, azathioprine, methotrexate, and/or intravenous immunoglobulin G [IVIgG]). Clinical tests conducted on all patients at the Neuromuscular Clinic included monopolar needle EMG, muscle biopsy, and knee extension (KE) strength test by quantitative isokinetic dynamometry (Biodex, Shirley, NY, USA) and Medical Research Council (MRC) muscle strength grading. Before treatment, all patients had abnormal EMG findings (fibrillations, positive sharp waves, and early recruiting motor unit action potentials). In addition, patients had to have a muscle biopsy that showed an inflammatory and/or necrotizing myopathy. Exclusion criteria included known muscular dystrophy; congenital, endocrine, or metabolic myopathy; or a preexisting inflammatory myopathy. A total of 26 patients met our inclusion/exclusion criteria for having a SAM during or after statin therapy.

\section{Biopsy}

Muscle biopsy samples were taken from the left or right vastus lateralis $(N=26)$, and if contraindicated (e.g., due to extreme atrophy), the right deltoid $(N=1)$. The biopsy was obtained using a Bergström needle, as described previously. ${ }^{36}$ Muscle biopsy tissues were placed on a cork, rapidly frozen in liquid nitrogen, and stored at $-85^{\circ} \mathrm{C}$. Seven-micron-thick cryostat sections were routinely stained with hematoxylin and eosin (H\&E), periodic acid-Schiff reaction, Oil Red O, and modified Gomori trichrome for assessment of morphology. A standard set of histochemical reactions was performed for the following enzymes: ATPase at $\mathrm{pH}$ 4.3, 4.5, and 10.0; alkaline and acid phosphatase; NADH; succinate dehydrogenase; myoadenylate deaminase; myophosphorylase; nonspecific esterase; and cytochrome $c$ oxidase. ${ }^{37}$ In all cases, biopsy material was cut to exhaustion, if required, until pathological changes were either detected or excluded. For identification of inflammatory cells, monoclonal antibodies against CD3 (Cell Marque, Rocklin, CA, USA), CD4 (Vector Laboratories, Burlingame, CA, USA), CD8, CD20, CD45, and CD68 (all from Daco, Carpenteria, CA, USA) were applied in recommended dilutions. Streptavidin-biotin detection system (Histostain Plus; Invitrogen, Grand Island, NY, USA) was used to visualize the immune reactions and followed by light hematoxylin counterstain.

\section{Diagnosis}

Clinical diagnostic criteria followed for PM and DM were based on those revised in a European Neuromuscular Centre Workshop in $2003,{ }^{38}$ themselves based on those initially set out by Bohan and Peter $^{39}$ in their seminal paper. Clinical diagnostic criteria for IMNM were from the same workshop. 
(a)

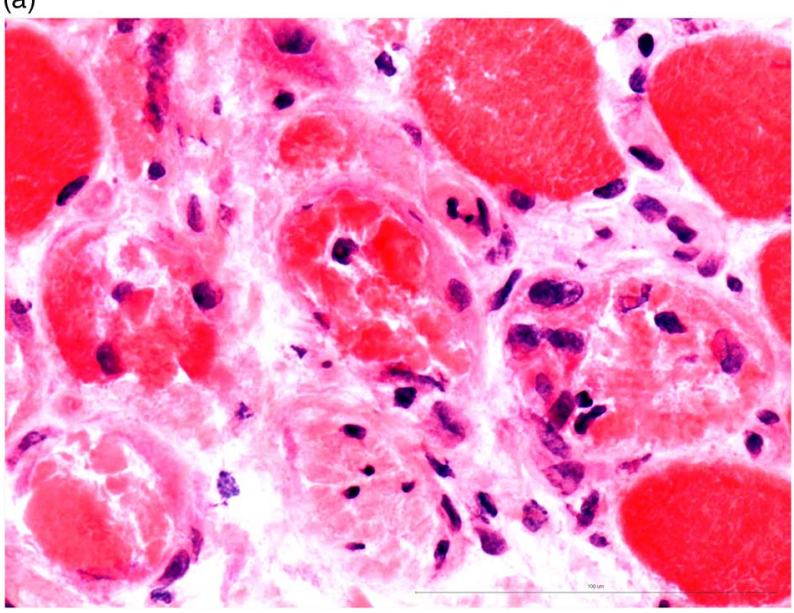

Figure 1a: Necrotizing myopathy. Disintegration of the muscle fibre and infiltration by macrophages. H\&E stain. Scale bar measures $100 \mu \mathrm{m}$.

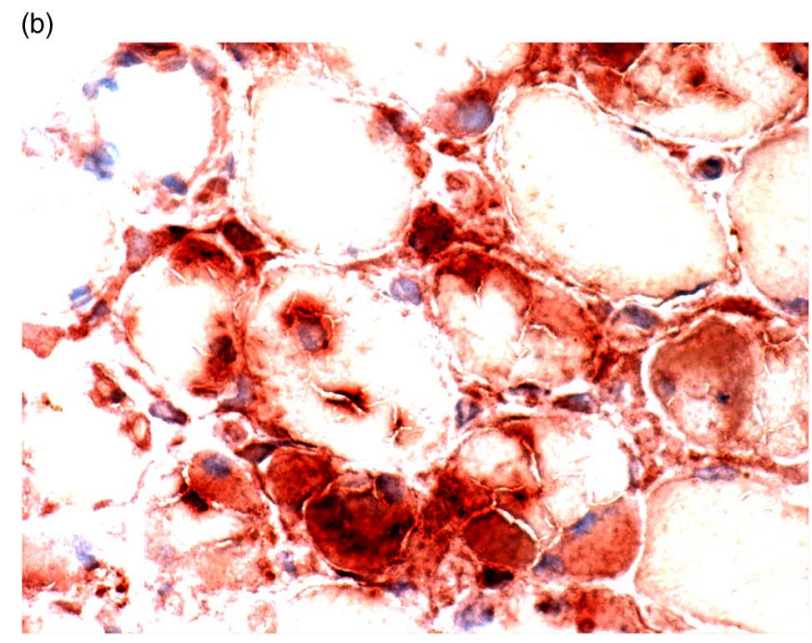

Figure 1b: Necrotizing myopathy. Strong acid phosphatase reaction in macrophages and necrotic or degenerating muscle fibres.

In brief, these IMNM diagnostic criteria consisted of proximal weakness, hyperCKemia, EMG showing irritable membrane instability, and biopsy showing prominent necrotic muscle fibres with no or minimal inflammation (Figures 1a and 1b). The histological criteria recommended by Hohlfeld ${ }^{40}$ were followed for the differential diagnosis of inflammatory myopathies. The presence of $\mathrm{T}$ cells in direct contact with nonnecrotic muscle fibres was obligatory for the diagnosis of PM (Figures 2a and $2 \mathrm{~b}$ ). A diagnosis of DM was based on the perivascular distribution of inflammatory cells, almost exclusively CD20 ${ }^{+}$ $\mathrm{B}$ cells with a few macrophages, with or without perifascicular atrophy. Biopsies with inflammatory infiltrates that did not strictly meet above requirements were designated as NSIM. All biopsies were reviewed twice by trained neuropathologists (B.L., J.P.P.) with more than 40 years of combined experiencefirst at initial diagnosis and again at data compilation following agreed-upon histological criteria and nomenclature for study purposes.

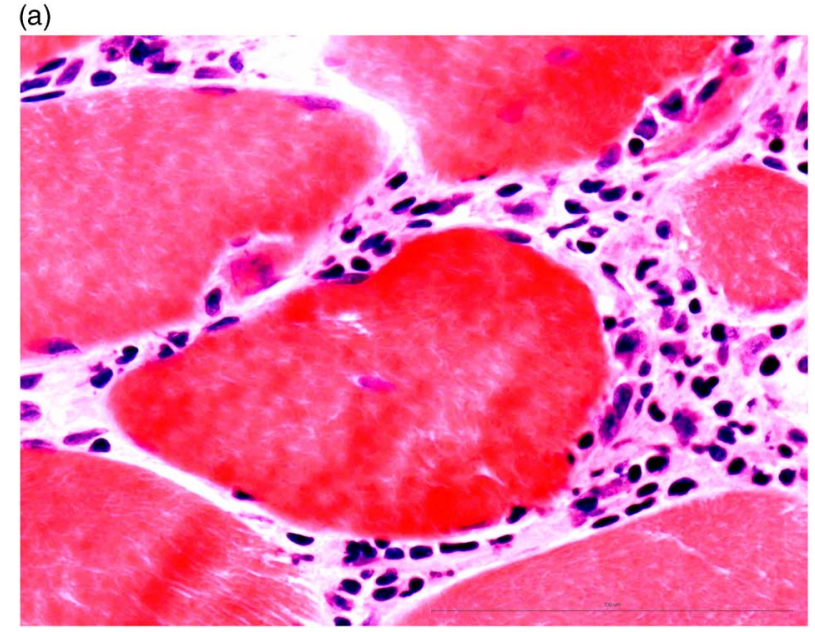

Figure 2a: Mononuclear inflammatory infiltration around intact muscle fibre in a patient with PM. Scale bar measures $100 \mu \mathrm{m}$.

(b)

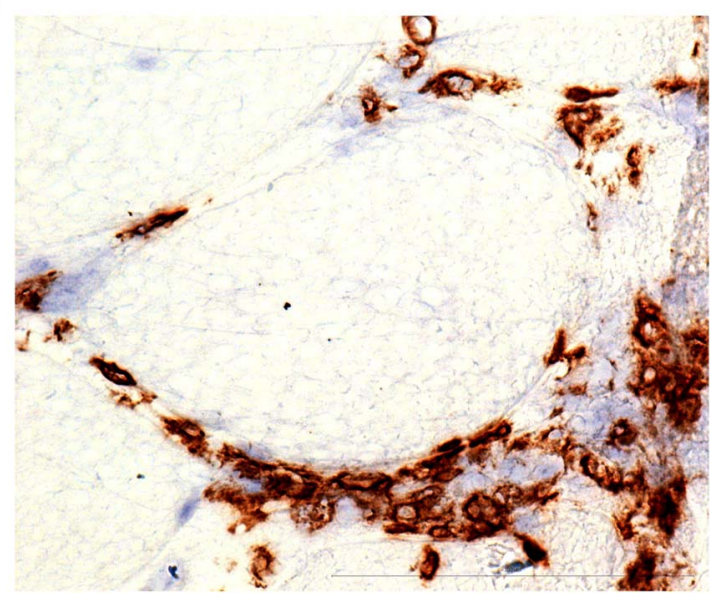

Figure 2b: Exclusive CD8-positive T-cells infiltration in deeper sections of the same biopsy sample. Scale bar measures $100 \mu \mathrm{m}$.

\section{Analysis}

To explore the hypothesis that statin-associated PM and IMNM are not completely separate entities but on the same pathophysiological spectrum, we compared characteristics of patients showing significant inflammation in the muscle biopsy (i.e., PM, NSIM) with patients diagnosed with IMNM (Table 1). The interval data of the groups was compared using two-tailed Student's $t$ test, and binary (yes-no) data were compared using Fisher's exact test (two-tailed $p$ value). Significant differences between the groups are noted in "Results." We performed another analysis in which we compared incidence of SAM at our clinic with that in other epidemiological reports. The estimated incidence at our clinic was based on the assumption that we see about two-thirds of the 1.4 million residents in our catchment area. Finally, we analyzed exposure to statins between patients with SAM seen at our clinic with those in the general public. Data from the general public were retrieved from the "Health, United States, 2010" report from the National Center for Health Statistics. ${ }^{41}$ 


\section{Table 1: SAM patients summary data}

\begin{tabular}{|c|c|c|c|}
\hline Demographics and pre-Tx clinical characteristics & Overall $(n=26)$ & Infl. group $(n=13)^{*}$ & IMNM group $(n=8) *$ \\
\hline Female sex & $37 \%$ & $62 \%$ & $13 \%$ \\
\hline Age at onset, mean (years) & $66.1(\mathrm{SD}=9.6)$ & 64.4 & 66.1 \\
\hline Max CK, mean (IU/L) & $6451(\mathrm{SD}=5744)$ & 3498 & 7751 \\
\hline $\mathrm{CK}$ at presentation, mean (IU/L) & $4105(\mathrm{SD}=3342)$ & 2320 & $5042(p<0.05)^{\frac{\ddagger}{7}}$ \\
\hline KE strength, mean (MRC) ${ }^{\dagger}$ & $4.25(\mathrm{SD}=0.54)$ & 4.40 & 4.09 \\
\hline Abnormal EMG & $100 \%$ & $100 \%$ & $100 \%$ \\
\hline Myalgias & $38 \%$ & $46 \%$ & $25 \%$ \\
\hline Weakness & $100 \%$ & $100 \%$ & $100 \%$ \\
\hline Dysphagia & $19 \%$ & $15 \%$ & $13 \%$ \\
\hline Rhabdomyolysis & $12 \%$ & $0 \%$ & $38 \%(p<0.05)^{\ddagger}$ \\
\hline Hospitalization & $23 \%$ & $8 \%$ & $38 \%$ \\
\hline Hypothyroidism & $19 \%$ & $23 \%$ & $25 \%$ \\
\hline Paraneoplastic & $4 \%$ & $8 \%$ & $0 \%$ \\
\hline Hypertension & $54 \%$ & $54 \%$ & $50 \%$ \\
\hline Diabetes mellitus type 2 & $62 \%$ & $54 \%$ & $50 \%$ \\
\hline Previous surgery & $35 \%$ & $31 \%$ & $25 \%$ \\
\hline Smoking & $23 \%$ & $23 \%$ & $25 \%$ \\
\hline Alcohol consumption & $65 \%$ & $62 \%$ & $75 \%$ \\
\hline \multicolumn{4}{|l|}{ Medication history } \\
\hline Atorvastatin & $81 \%$ & $69 \%$ & $88 \%$ \\
\hline Rosuvastatin & $35 \%$ & $31 \%$ & $25 \%$ \\
\hline Simvastatin & $4 \%$ & $0 \%$ & $13 \%$ \\
\hline Unknown statin & $8 \%$ & $15 \%$ & $0 \%$ \\
\hline Multiple statins & $23 \%$ & $8 \%$ & $25 \%$ \\
\hline Ezetimibe & $31 \%$ & $23 \%$ & $50 \%$ \\
\hline Cholestyramine & $4 \%$ & $0 \%$ & $13 \%$ \\
\hline Statin-interacting drugs & $23 \%$ & $38 \%$ & $13 \%$ \\
\hline \multicolumn{4}{|l|}{ LLD rechallenge instances } \\
\hline Rosuvastatin [adverse outcomes] & $8[4]$ & & \\
\hline Atorvastatin [adverse outcomes] & $3[2]$ & & \\
\hline Pravastatin [adverse outcomes] & $1[1]$ & & \\
\hline Ezetimibe [adverse outcomes] & $12[3]$ & & \\
\hline Cholestyramine [adverse outcomes] & $1[0]$ & & \\
\hline Fenofibrate [adverse outcomes] & $1[1]$ & & \\
\hline Relapse after IS therapy taper & $67 \%$ & $63 \%$ & $75 \%$ \\
\hline \multicolumn{4}{|l|}{ Post-Tx clinical characteristics } \\
\hline $\mathrm{CK}$, mean $(\mathrm{IU} / \mathrm{L})$ & $694(\mathrm{SD}=961)$ & 594 & 749 \\
\hline KE strength, mean (MRC) ${ }^{\dagger}$ & $4.7(\mathrm{SD}=0.4)$ & 4.8 & $4.4(p<0.05)^{\ddagger}$ \\
\hline
\end{tabular}

$\mathrm{Tx}=$ treatment; Infl. = inflammation; $\mathrm{SD}=$ standard deviation; $\mathrm{CK}=$ creatine kinase; $\mathrm{KE}=$ knee extension; $\mathrm{MRC}=$ Medical Research Council;

LLD = lipid-lowering drug; IS therapy = immunosuppressive therapy.

*To explore the hypothesis of PM and IMNM being a single entity, we compared characteristics of patients who had diagnoses involving biopsies showing significant inflammation (i.e., PM, NSIM; patient nos. 9-20 and 26 in Table 2) with patients who had a diagnosis of IMNM (patient nos. 1-8 in Table 2). Sum of the number of patients in both groups is not the total of 26 because patients with overlapping distinct diagnoses are not included in either group.

"The semigrades of MRC strength scale are converted as follows: "5" $=5.0 ; " 5-"=4.7 ; " 4+"=4.3 ; " 4 "=4.0 ; " 4-"=3.7$; " $3+"=3.3$; etc.

${ }^{\ddagger}$ To calculate the $p$ values, we compared the interval data of the groups by using two-tailed Student's $t$ test, and we compared the binary (yes-no) data by using Fisher's exact test (two-tailed $p$ value). 


\section{RESUlTS}

We studied 26 patients, consisting of 17 men and 9 women. Their characteristics are summarized in Table 1; detailed casespecific data are shown in Tables $2 \mathrm{a}$ and $2 \mathrm{~b}$. The average age of onset was 66.4 years (range, 47-81 years). For SAM diagnoses; ten patients had PM, eight had IMNM, three had a NSIM, and five had changing inflammatory myopathy designations (i.e., these patients had more than one biopsy with different diagnoses). Risk factors associated with acute statin myopathies ${ }^{42}$ were also associated with SAMs, including age, ${ }^{43}$ hypothyroidism, diabetes mellitus type 2, previous surgery, and alcohol consumption (see Table 1).
Table 1 summarizes the proportion of patients exposed to certain statins (which exceeds $100 \%$ because some patients were exposed to multiple statins). Average duration of treatment with statins before symptom onset was 72.4 months. In addition to statin exposure, eight patients had also taken ezetimibe, and one had taken cholestyramine as other LLDs. Six patients were concomitantly taking other drugs that amplify statin myotoxicity or interact with statin metabolism, namely, amiodarone, amlodipine, omeprazole, venlafaxine, and clarithromycin. Patients who concomitantly took these drugs had a paradoxically lower presenting CK $(2,259$ IU/L to 4,694 IU/L, $p=0.023)$ and a lower posttreatment CK (204 IU/L to $842 \mathrm{IU} / \mathrm{L}, p=0.019)$. All patients were

Table 2a: Patient-specific data

\begin{tabular}{|c|c|c|c|c|c|c|}
\hline $\begin{array}{l}\text { Case \#/sex/ } \\
\text { age at onset }\end{array}$ & Diagnosis(es) & $\begin{array}{l}\text { Statin(s), dose (mg), [duration before } \\
\text { Neuromuscular Clinic exam]* }\end{array}$ & $\begin{array}{l}\text { Statin duration } \\
\text { before onset* }\end{array}$ & $\begin{array}{l}\text { Duration off } \\
\text { statins before first } \\
\mathbf{B x}\end{array}$ & $\begin{array}{l}\text { Max pre-Tx CK and } \\
\text { post-Tx CK (IU/L) }\end{array}$ & $\begin{array}{l}\text { Pre- and post-Tx KE } \\
\text { strength (MRC) }\end{array}$ \\
\hline No. $1 / \mathrm{M} / 68$ & IMNM & Atosn $20[4 y 5 \mathrm{~m}]$ & $4 \mathrm{y} 4 \mathrm{~m}$ & $0 \mathrm{y} 0 \mathrm{~m} 12 \mathrm{~d}$ & $5295 \rightarrow 208$ & $4 \rightarrow 5$ \\
\hline No. $2 / \mathrm{M} / 63$ & IMNM & Rossn $20[0 \mathrm{y} 8 \mathrm{~m}]$ & $0 \mathrm{y} 7 \mathrm{~m}$ & $0 \mathrm{y} 0 \mathrm{~m} 15 \mathrm{~d}$ & $2274 \rightarrow 571$ & $3+\rightarrow 4+$ \\
\hline No. $3 / \mathrm{M} / 58$ & IMNM & Atosn $[6 y \mathrm{y}]$ & $6 y 4 \mathrm{~m}$ & $3 y 1 \mathrm{~m}$ & $4240 \rightarrow 825$ & $4 \rightarrow \mathrm{N} / \mathrm{A}$ \\
\hline No. $4 / \mathrm{M} / 62$ & IMNM & Atosn $[2 \mathrm{y} 0 \mathrm{~m}]$ & $0 \mathrm{y} 1 \mathrm{~m}$ & $2 \mathrm{y} 2 \mathrm{~m}$ & $5000 \rightarrow 1194$ & $4+\rightarrow 4+$ \\
\hline No. $5 / M / 81$ & IMNM & Atosn $80[1 \mathrm{y} 7 \mathrm{~m}]$ & $1 \mathrm{y} 7 \mathrm{~m}$ & $0 y 2 \mathrm{~m}$ & $10000 \rightarrow 79$ & $\mathrm{~N} / \mathrm{A} \rightarrow \mathrm{N} / \mathrm{A}$ \\
\hline No. $6 / \mathrm{M} / 56$ & IMNM & Atosn [1y $11 \mathrm{~m}]$ & $0 \mathrm{y} 1 \mathrm{~m}$ & $0 \mathrm{y} 6 \mathrm{~m}$ & $19000 \rightarrow 2666$ & $5 \rightarrow 4+$ \\
\hline No. $7 / F / 78$ & IMNM & Atosn [19y 1m], Rossn 40 [1y 4m] & $20 y 3 m$ & $0 \mathrm{y} 1 \mathrm{~m}$ & $4200 \rightarrow 23$ & $4 \rightarrow 4$ \\
\hline No. $8 / \mathrm{M} / 73$ & $\begin{array}{l}\text { IMNM(Bx1), } \\
\text { IMNM(Bx2) }\end{array}$ & Simsn [2y 0m], Atosn [12y 2m] & $14 \mathrm{y} 0 \mathrm{~m}$ & $0 y 2 \mathrm{~m}$ & $12000 \rightarrow 500$ & $4 \rightarrow 4-$ \\
\hline No. $9 / \mathrm{M} / 57$ & PM & Unknown "statin" reported & N/A & N/A & $3831 \rightarrow 500$ & $5 \rightarrow 5$ \\
\hline No. $10 / \mathrm{F} / 56$ & PM & Atosn 10 [“few"y] & "few"y & N/A & $946 \rightarrow 128$ & $5 \rightarrow$ N/A \\
\hline No. $11 / \mathrm{F} / 77$ & PM & Atosn $40[24 \mathrm{y} 9 \mathrm{~m}]$, Atosn $80[0 \mathrm{y} 4 \mathrm{~m}]$ & $25 \mathrm{y} 0 \mathrm{~m}$ & $0 \mathrm{y} 1 \mathrm{~m}$ & $704 \rightarrow 117$ & $4 \rightarrow \mathrm{N} / \mathrm{A}$ \\
\hline No. $12 / F / 58$ & PM & Atosn $[5 \mathrm{y} 0 \mathrm{~m}]$ & $4 y 7 \mathrm{~m}$ & $0 \mathrm{y} 2 \mathrm{~m}$ & $7000 \rightarrow 42$ & $\mathrm{~N} / \mathrm{A} \rightarrow 4$ \\
\hline No. $13 / \mathrm{M} / 59$ & PM & Atosn $10[4 \mathrm{y} 6 \mathrm{~m}]$ & $4 y 6 \mathrm{~m}$ & $2 \mathrm{y} 3 \mathrm{~m}$ & $8200 \rightarrow 4156$ & $5 \rightarrow 5$ \\
\hline No. $14 / F / 75$ & PM & Unknown "statin" reported & N/A & N/A & $3741 \rightarrow 344$ & $4 \rightarrow 4+$ \\
\hline No. $15 / F / 71$ & PM & Rossn [1y 7m] & $1 \mathrm{y} 0 \mathrm{~m}$ & $0 \mathrm{y} 7 \mathrm{~m}$ & $974 \rightarrow 190$ & $4 \rightarrow 4+$ \\
\hline No. $16 / \mathrm{M} / 55$ & PM & Atosn $[0 \mathrm{y} 1 \mathrm{~m}]$ & N/A & $0 \mathrm{y} 6 \mathrm{~m}$ & $1187 \rightarrow \mathrm{N} / \mathrm{A}$ & $4+\rightarrow 5$ \\
\hline No. $17 / \mathrm{F} / 47$ & PM & Rossn 10 [3y 8m], Rossn [1y 0m] & N/A & $0 \mathrm{y} 6 \mathrm{~m}$ & $1656 \rightarrow 187$ & $4 \rightarrow 5$ \\
\hline No. $18 / \mathrm{F} / 70$ & NSIM & Atosn 10 [8y $5 \mathrm{~m}]$ & N/A & $0 \mathrm{y} 0 \mathrm{~m} 5 \mathrm{~d}$ & $6574 \rightarrow 84$ & $4-\rightarrow 5$ \\
\hline No. $19 / \mathrm{M} / 80$ & NSIM & Atosn 20 [3y 9m] & $3 y 9 \mathrm{~m}$ & 0y $3 \mathrm{~m}$ & $1437 \rightarrow 102$ & $5 \rightarrow 5$ \\
\hline No. $20 / F / 60$ & NSIM & Atosn [0y 0m 3d], Rossn [0y 1m] & $0 \mathrm{y} 1 \mathrm{~m}$ & $0 \mathrm{y} 9 \mathrm{~m}$ & $3228 \rightarrow 1031$ & $5 \rightarrow 5$ \\
\hline No. $21 / \mathrm{M} / 63$ & $\begin{array}{l}\mathrm{IMNM}(\mathrm{Bx} 1), \mathrm{PM} \\
\quad(\mathrm{Bx} 2)\end{array}$ & Atosn [0y $2 \mathrm{~m}]$, Rossn $[0 \mathrm{y} 2 \mathrm{~m}]$ & $0 y 5 \mathrm{~m}$ & $0 y 5 \mathrm{~m}$ & $13000 \rightarrow 1900$ & $4-\rightarrow 5$ \\
\hline No. $22 / \mathrm{M} / 77$ & $\begin{array}{l}\mathrm{IMNM}(\mathrm{Bx} 1), \mathrm{PM} \\
\quad(\mathrm{Bx} 2)\end{array}$ & Atosn $10[4 \mathrm{y} 8 \mathrm{~m}]$ & $4 y 3 \mathrm{~m}$ & Still on statin & $7913 \rightarrow 439$ & $4-\rightarrow 5$ \\
\hline No. $23 / \mathrm{M} / 81$ & $\begin{array}{l}\mathrm{IMNM}(\mathrm{Bx} 1), \mathrm{PM} \\
\quad(\mathrm{B} 2)\end{array}$ & Atosn [5y 0m], Rossn 5 [5y 4m] & $9 \mathrm{y} 6 \mathrm{~m}$ & $5 \mathrm{y} 3 \mathrm{~m}$ & $13000 \rightarrow \mathrm{N} / \mathrm{A}$ & $4-\rightarrow 5$ \\
\hline No. $24 / \mathrm{M} / 73$ & $\begin{array}{l}\mathrm{IMNM}(\mathrm{Bx} 1) \\
\quad \mathrm{NSIM}(\mathrm{Bx} 2)\end{array}$ & Atosn 80 [6y $5 \mathrm{~m}]$ & $6 y 6 \mathrm{~m}$ & $0 y 3 \mathrm{~m}$ & $2730 \rightarrow 524$ & $4+\rightarrow 4$ \\
\hline No. $25 / \mathrm{M} / 60$ & $\begin{array}{l}\text { IMNM(Bx1), } \\
\text { NSIM(Bx2) }\end{array}$ & Atosn $[2 \mathrm{y} 5 \mathrm{~m}]$ & N/A & $1 \mathrm{y} 1 \mathrm{~m}$ & $23600 \rightarrow 1340$ & $5 \rightarrow 5$ \\
\hline No. $26 / \mathrm{M} / 72$ & $\begin{array}{l}\text { Normal(Bx1), PM } \\
\quad(\mathrm{B} 2), \mathrm{PM}(\mathrm{Bx} 3)\end{array}$ & Atosn $[0 \mathrm{y} 0 \mathrm{~m} 7 \mathrm{~d}]$ & N/A & $0 \mathrm{y} 4 \mathrm{~m}$ & $6000 \rightarrow 407$ & $4 \rightarrow 5$ \\
\hline
\end{tabular}

$\mathrm{M}=$ male; $\mathrm{F}=$ female; $\mathrm{Bx}=$ biopsy; Norm. = normal; Atosn = atorvastatin; Rossn = rosuvastatin; Simsn = simvastatin; Lovsn = lovastatin; LLD = lipidlowering drug; Eze = ezetimibe; $\mathrm{Tx}=$ treatment; $\mathrm{KE}=$ knee extension; $\mathrm{MRC}=$ Medical Research Council scale.

${ }^{*}$ Time durations are given in the format [number of years]y [number of months]m [number of days]d. For instance, " $4 \mathrm{y} 5 \mathrm{~m}$ " means 4 years and 5 months 
Table 2b: Patient-specific data (continued)

\begin{tabular}{|c|c|c|c|c|c|c|}
\hline $\begin{array}{l}\text { Case \#/sex/ } \\
\text { age at onset }\end{array}$ & Diagnosis(es) & $\begin{array}{l}\text { Concomitant interacting } \\
\text { drugs (incl. LLDs) }\end{array}$ & $\begin{array}{l}\text { Immunosup- } \\
\text { pressants (IS) used }\end{array}$ & $\begin{array}{l}\text { IS Tx } \\
\text { duration* }\end{array}$ & $\begin{array}{l}\text { Relapse after } \\
\text { IS taper? }\end{array}$ & $\begin{array}{l}\text { LLD rechallenge, dose (mg), [adverse effects } \\
\text { if applicable] }\end{array}$ \\
\hline No. $1 / \mathrm{M} / 68$ & IMNM & & Pred, Mtx, IVIgG & $>4 y 5 \mathrm{~m}$ & Yes & Eze 10 \\
\hline No. $2 / \mathrm{M} / 63$ & IMNM & Eze & $\begin{array}{l}\text { Pred, Mtx, Aza, } \\
\text { IVIgG }\end{array}$ & $>0 \mathrm{y} 10 \mathrm{~m}$ & Yes & \\
\hline No. $3 / \mathrm{M} / 58$ & IMNM & Eze & Mtx, Aza, IVIgG & $>1 \mathrm{y} 4 \mathrm{~m}$ & N/A & Eze \\
\hline No. $4 / \mathrm{M} / 62$ & IMNM & Eze & Pred, Mtx & $>1 \mathrm{y} 2 \mathrm{~m}$ & N/A & Eze \\
\hline No. $5 / \mathrm{M} / 81$ & IMNM & Amlodipine & Pred & 0y $5 \mathrm{~m}$ & No & Eze 10 [weakness, dysphagia] \\
\hline No. $6 / \mathrm{M} / 56$ & IMNM & & Pred, Mtx & $>0 \mathrm{y} 6 \mathrm{~m}$ & N/A & \\
\hline No. $7 / F / 78$ & IMNM & & Pred & 0y $5 \mathrm{~m}$ & No & Rossn 40 [rhabdomyolysis] \\
\hline No. $8 / \mathrm{M} / 73$ & $\begin{array}{l}\operatorname{IMNM}(\mathrm{Bx} 1) \\
\operatorname{IMNM}(\mathrm{Bx} 2)\end{array}$ & & Pred, Mtx, IVIgG & $>4 \mathrm{y} 4 \mathrm{~m}$ & No & $\begin{array}{l}\text { Atosn [hyperCKemia, weakness], Eze, Pstn } \\
40 \text { [hyperCKemia, weakness], Cho }\end{array}$ \\
\hline No. 9/M/57 & PM & & Pred, Mtx & $>1 \mathrm{y} 1 \mathrm{~m}$ & N/A & \\
\hline No. $10 / \mathrm{F} / 56$ & $\mathrm{PM}$ & Nortriptyline, omeprazole & $\begin{array}{l}\text { Pred, Mtx, Mpre, } \\
\text { Aza, IVIgG }\end{array}$ & $5 \mathrm{y} 5 \mathrm{~m}$ & Yes & Eze 10 \\
\hline No. $11 / F / 77$ & $\mathrm{PM}$ & Eze & Pred, IVIgG & $1 \mathrm{y} 11 \mathrm{~m}$ & Yes & $\begin{array}{l}\text { Atosn } 80 \text { (from 40) + Eze [fatigue, weakness], } \\
\text { Rossn 2.5, Rossn } 5\end{array}$ \\
\hline No. $12 / F / 58$ & $\mathrm{PM}$ & & Pred, Mtx & $>0 \mathrm{y} 9 \mathrm{~m}$ & N/A & Eze 10 \\
\hline No. $13 / \mathrm{M} / 59$ & $\mathrm{PM}$ & & Pred, Mtx & $>0 \mathrm{y} 7 \mathrm{~m}$ & N/A & \\
\hline No. $14 / F / 75$ & $\mathrm{PM}$ & $\begin{array}{r}\text { Eze, venlafaxine, } \\
\text { clarithromycin }\end{array}$ & Pred, Mtx, IVIgG & $>5 \mathrm{y} 8 \mathrm{~m}$ & Yes & \\
\hline No. $15 / F / 71$ & $\mathrm{PM}$ & & Pred, Mtx & $>2 \mathrm{y} 1 \mathrm{~m}$ & No & \\
\hline No. $16 / \mathrm{M} / 55$ & $\mathrm{PM}$ & & Pred & $0 \mathrm{y} 0 \mathrm{~m} 14 \mathrm{~d}$ & No & \\
\hline No. $17 / F / 47$ & $\mathrm{PM}$ & Omeprazole & Mtx & $>1 \mathrm{y} 6 \mathrm{~m}$ & Yes & Rossn [myalgias, weakness] \\
\hline No. $18 / \mathrm{F} / 70$ & NSIM & & Pred & $>3 \mathrm{y} 0 \mathrm{~m}$ & N/A & Ffib [myalgias, hyperCKemia] \\
\hline No. $19 / \mathrm{M} / 80$ & NSIM & Amlodipine, doxepin & Pred, Mtx & $>0 \mathrm{y} 5 \mathrm{~m}$ & N/A & \\
\hline No. $20 / \mathrm{F} / 60$ & NSIM & & Pred, Mtx & $>1 \mathrm{y} 6 \mathrm{~m}$ & Yes & Rossn [stomach problems] \\
\hline No. $21 / \mathrm{M} / 63$ & $\begin{array}{l}\mathrm{IMNM}(\mathrm{Bx} 1), \mathrm{PM} \\
\quad(\mathrm{Bx} 2)\end{array}$ & Eze & Pred, Mtx & $>1$ y $8 \mathrm{~m}$ & N/A & Rossn, Eze [hyperCKemia] \\
\hline No. 22/M/77 & $\begin{array}{l}\mathrm{IMNM}(\mathrm{Bx} 1), \mathrm{PM} \\
\quad(\mathrm{Bx} 2)\end{array}$ & & Pred, Mtx & $>1 \mathrm{y} 6 \mathrm{~m}$ & N/A & \\
\hline No. $23 / \mathrm{M} / 81$ & $\begin{array}{l}\mathrm{IMNM}(\mathrm{Bx} 1), \mathrm{PM} \\
\quad(\mathrm{Bx} 2)\end{array}$ & Amlodipine & Pred, Aza, IVIgG & $>0 \mathrm{y} 10 \mathrm{~m}$ & N/A & Rossn 5 \\
\hline No. $24 / \mathrm{M} / 73$ & $\begin{array}{l}\text { IMNM(Bx1), NSIM } \\
(\mathrm{Bx} 2)\end{array}$ & & Pred & $>1 \mathrm{y} 11 \mathrm{~m}$ & Yes & Eze, Rossn [weakness, falls, hyperCKemia] \\
\hline No. $25 / \mathrm{M} / 60$ & $\begin{array}{l}\text { IMNM(Bx1), NSIM } \\
(\mathrm{Bx} 2)\end{array}$ & Rosiglitazone & $\begin{array}{l}\text { Pred, Mtx, Aza, } \\
\text { IVIgG }\end{array}$ & $>2 \mathrm{y} 4 \mathrm{~m}$ & Yes & \\
\hline No. 26/M/72 & $\begin{array}{l}\text { Normal(Bx1), PM } \\
\quad(\mathrm{B} \times 2), \mathrm{PM}(\mathrm{B} \times 3)\end{array}$ & Amlodipine & Pred, Mtx & $>2 \mathrm{y} 0 \mathrm{~m}$ & No & Eze 10, Atosn 20 \\
\hline
\end{tabular}

$\mathrm{M}=$ male; $\mathrm{F}=$ female; $\mathrm{Bx}=$ biopsy; Atosn = atorvastatin; Rossn = rosuvastatin; Simsn = simvastatin; Lovsn = lovastatin; LLD = lipid-lowering drug; Eze = ezetimibe; $\mathrm{Tx}=$ treatment; $\mathrm{KE}=$ knee extension; $\mathrm{IS}=$ immunosuppressants; Pred = prednisone; Mtx = methotrexate; IVIgG = intravenous immunoglobulin G; Aza = azathioprine; Mpre = methylprednisolone; Ffib = fenofibrate.

${ }^{*}$ Time durations are given in the format [number of years]y [number of months]m [number of days]d. For instance, “4y $5 \mathrm{~m} "$ means 4 years and 5 months

initially found to have hyperCKemia and weakness in at least one major muscle group. Also, males showed a higher peak CK $(8,159 \mathrm{IU} / \mathrm{L}$ to $3,302 \mathrm{IU} / \mathrm{L}, p=0.0088)$ and higher posttreatment CK (950 IU/L to $223 \mathrm{IU} / \mathrm{L}, p=0.023)$ compared with females.

Thirty-seven percent of patients showed some improvement after medication discontinuation (i.e., diminished myalgias and/or weakness and/or serum CK levels); however, all patients ultimately ended up with progressive or persisting symptoms of weakness, hyperCKemia, and/or myalgias. One patient also developed distal weakness, and $23 \%$ had to be hospitalized owing to severity of symptoms.

Average time off statins before an initial biopsy diagnosis of IMNM was 12.0 months, and for an initial biopsy diagnosis of PM/NSIM was 6.6 months (see Table 2a). Seven patients underwent multiple biopsies: six with two biopsies and one with three biopsies. The reasons for repeat biopsies included deciphering the 


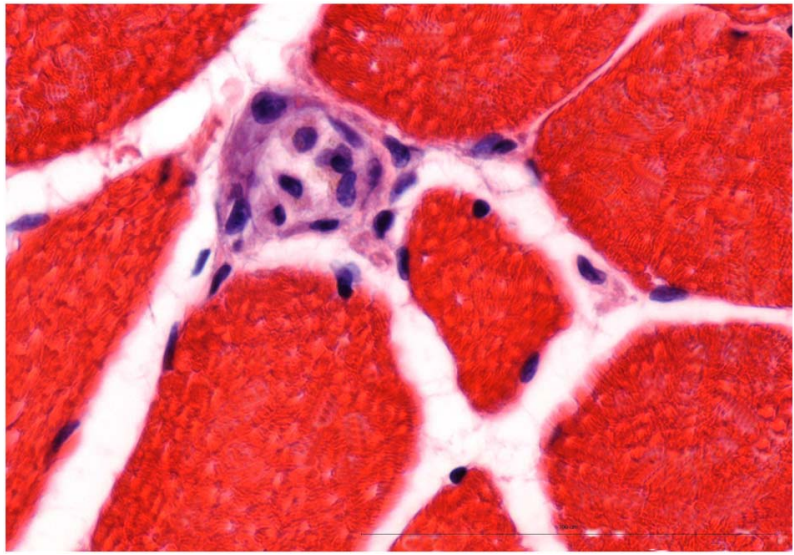

Figure 3: First biopsy of 77-year-old patient (no. 22) on statin for 4 years and 8 months. Necrotizing myopathy without inflammation. Necrotic fibre infiltrated by macrophages. $H \& E$ stain. Scale bar measures $100 \mu \mathrm{m}$.

cause for worsening symptoms despite therapy and confirming diagnosis. Average time between first and second biopsy was 75.8 months. Time between second and third biopsy for the single patient was 35.1 months. Five of these seven patients had a transformation from either IMNM (myofibre necrosis) to an inflammatory myopathy or vice versa. For an example, we have depicted the histological images of such a transformation in patient No. 22. Figure 3 shows the first biopsy, which provided a diagnosis of IMNM, and Figures $4 \mathrm{a}$ and $4 \mathrm{~b}$ show the second biopsy, performed 6 months later, which gave a diagnosis of PM. Five of these seven patients had subsequent biopsies in a different anatomical location from the first. Four of the five had changing histological diagnoses.

All patients were treated with immunosuppressive therapy with a good response: lowering serum CK levels (100\%) and improving or maintaining an MRC KE strength of at least $4+(86 \%)$. Mean duration on immunosuppressive therapy at data collection was 23.4 months, with each patient taking an average of 2.2 immunosuppressive agents, including prednisone (96\%), methotrexate (73\%), azathioprine (19\%), and IVIgG (35\%). Fifteen patients tried to lower the immunosuppressant dose, of which ten patients relapsed and required a return to a higher dose, whereas five patients tapered to a lower dose of prednisone (2.5-5.0 mg) without relapse (see Table 2b). On the basis of clinical need (hypercholesterolemia and risk factors), 10 of these 15 were rechallenged with other LLDs for a total of 19 rechallenge instances in this subgroup of those who tapered (some patients had more than one rechallenge). Within these 19 rechallenges, ten instances of adverse effects occurred: two of the three atorvastatin rechallenge instances, four of the six for rosuvastatin, one of the one for pravastatin, and two of the eight for ezetimibe (see Table 2b). Adverse effects generally included worsening hyperCKemia, weakness, falls, and accelerated decline, as well as one instance each of dysphagia and stomach problems.

We also conducted another analysis to estimate incidence of inflammatory myopathies not necessarily associated with statins within our clinic's catchment area. We found 46 cases of inflammatory myopathy (PM, DM and nonspecific myositis) over the observation period (2005-2011), and we see around two-thirds of the patients with neuromuscular problems in our 1.4 million-resident (a)

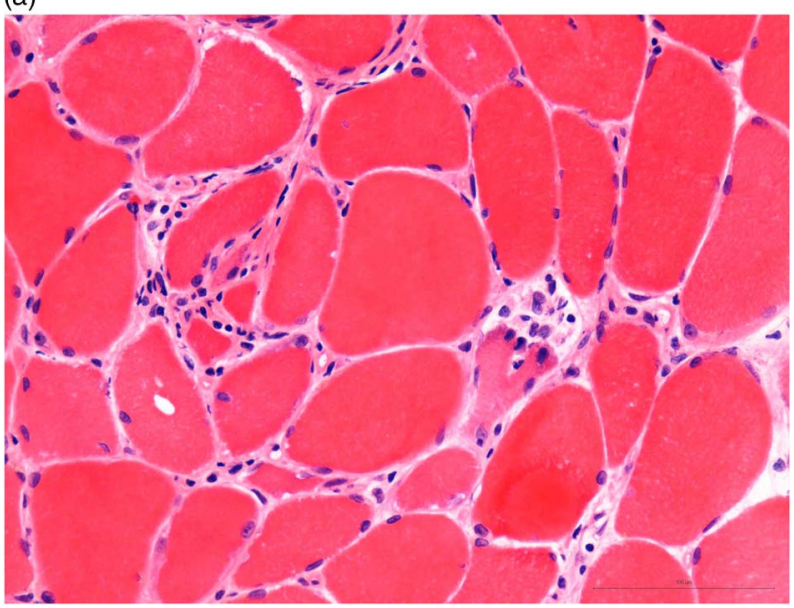

Figure 4a: Second biopsy of same 77 year-old patient (no. 22) performed six months later. Muscle fibres surrounded by a few macrophages. $H \& E$ stain. Scale bar measures $100 \mu \mathrm{m}$.

(b)

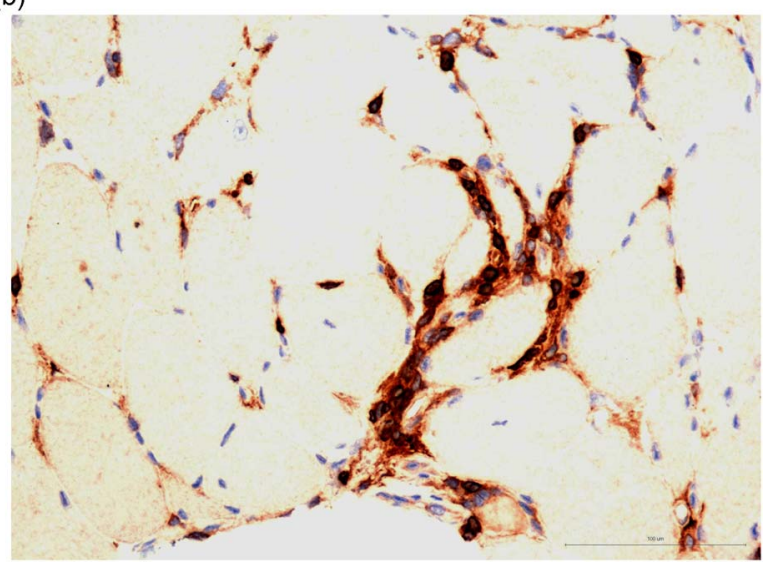

Figure 4b: Same biopsy as in Figure 4a. Muscle fibres surrounded or infiltrated by CD3-positive lymphocytes. CD3 immunostain. Scale bar measures $100 \mu \mathrm{m}$.

Abbreviations: HMGCR, 3-hydroxy-3-methylglutaryl coenzyme A reductase; $C K$, creatine kinase; SAM, statin-associated autoimmune myopathy; PM, typical polymyositis; DM, typical dermatomyositis; IMNM, immune-mediate necrotizing myopathy; NSIM, nonspecific inflammatory myopathy; LLD, lipid-lowering drug; EMG, electromyography; IVIgG, intravenous immunoglobulin $G$; MRC, Medical Research Council; KE, knee extension strength test; IBM, inclusion body myositis.

catchment area, so we calculated an expected incidence rate of 7.0 cases per million patients per year. This finding is very close to other reports, which cite $5-10^{44,45}$ and $2.2-8.8^{46}$ cases of inflammatory myopathies per million per year. We further analyzed the rate of statin usage in our population of inflammatory myopathy cases (excluding IBM, because little evidence for its association with statins exists) and found that a greater than expected $69 \%$ of these patients during this time took a statin, compared with the $25 \%$ rate in the U.S. population in the same age group (aged 45 years and older) ${ }^{41}$ further supporting that the association is more than coincidental. As expected, of our patients with IBM, only $20 \%$ were exposed to statins. Finally, in comparing statin-exposed patients that had inflammatory myopathy with statin-naïve patients, we observed 
two statistically significant differences: statin-naïve patients had lower pretreatment peak CK levels (2744 vs. 6451; $p=0.02)$ and were younger (53.2 vs. 66.1 years; $p=0.03$ ).

\section{DISCUSSION}

This study offers important clinical information regarding diagnosis and treatment of patients with SAMs and provides data suggesting that statin-associated IMNM and PM may be related to the same pathophysiological mechanism and part of a diagnostic spectrum. Patients with statin-associated PM met established criteria for "idiopathic" PM in all aspects, implying that some proportion of "idiopathic" PM cases may have some identifiable trigger.

Because statins are one of the most frequently prescribed drugs, ${ }^{2,3}$ we cannot exclude coincidence as the cause for the association between statin use and the observed myopathies. However, reports of immunomodulatory effects of statins are increasing, as are reports of the connection of this drug with other autoimmune diseases such as lupus and myasthenia gravis. ${ }^{47,48}$ We also found a higher rate of statin usage in our entire cohort of autoimmune myopathy patients-69\% compared with $25 \%$ in the general public - which supports data from previous studies that reported rates of $47.6 \%$ and $82 \%{ }^{27}$ of statin exposure in patients with inflammatory myopathy and IMNM, respectively. Finally, that $67 \%$ of all instances of statin rechallenge led to recurrence of signs and symptoms supports a true etiological association. Furthermore, several reports show that extended exposure to statins may induce the following: (1) increase of human leukocyte antigen class I expression by myocytes, ${ }^{28}$ (2) chronic exposure of latent antigens after myocyte damage, and (3) promotion of a Th2type immune response as seen in "idiopathic" PM and DM. ${ }^{50,51}$

Within our sample of 26 SAM cases, we found several clinical associations. The observation of higher CKs (both peak and after treatment) in males was expected because males have greater muscle mass, show a greater inflammatory response to muscle damage, ${ }^{52}$ and have higher CK levels in general. ${ }^{53}$ Next, a paradoxical relationship was found where patients that concomitantly took drugs that amplify statin myotoxicity had lower presenting and posttreatment CK levels. These drugs would be expected to exacerbate acute statin-associated myotoxicity, and so in our case, this strengthens the likelihood that SAMs are not merely a persistence of acute drug-associated myotoxicity. Patients' sexes may also have confounded this finding: the group taking interacting drugs had a male:female ratio of $1: 1$, whereas the group that did not had a ratio of 2.2:1. No associations were observed between age and measured variables, including severity of disease, comorbidities, or treatment given.

Throughout the statin therapy, 25 instances of LLD rechallenge occurred. We found no association between whether the patient experienced adverse effects during LLD rechallenge versus age or sex. Comparing statins against other LLDs, we found that statins seem to have more severe and a higher risk of adverse effects. The other LLDs, ezetimibe, cholestyramine, and fenofibrate, seemed to be relatively better tolerated, with fewer and milder effects, consisting of hyperCKemia and fluctuating weakness. This finding is in agreement with a previous study on LLD rechallenge after acute statin-associated myotoxicity. ${ }^{54}$ Patients who relapsed upon tapering immunosuppressive therapy were more likely to have had IVIgG therapy than those who did not relapse ( $73 \%$ to $17 \%, p=0.0498$ ). This outcome probably occurred because IVIgG was generally given to patients with more severe myopathy, and this association merely reflects that those with more severe disease were most likely to relapse upon tapering immunosuppressive therapy.

To investigate the hypothesis that IMNM is pathophysiologically related to PM, we tested the homogeneity of all measured factors between patients with IMNM and patients with significant inflammation on biopsy (i.e., PM and NSIM). The IMNM group showed more severe symptoms, consisting of lower posttreatment KE strength on the MRC scale (4.3 to $4.8, p=0.038$ ), as well as higher pretreatment CK $(5,042 \mathrm{IU} / \mathrm{L}$ to $2,321 \mathrm{IU} / \mathrm{L}, p=0.031)$. All other factors, including duration of statin treatment to onset, presenting symptoms, comorbidities, location of weakness, age, and sex were relatively homogenous between groups, offering preliminary evidence that the two groups do not represent distinct entities.

We hypothesize that IMNM and the statin-related inflammatory myopathies, PM and NSIM, are not separate entities but rather share a single pathophysiological spectrum. To reconcile the fact that not all statin-associated necrotizing myopathy patients develop inflammatory myopathy symptoms, we propose that a patient exposed to statins who develops myonecrosis may have either a myotoxicity that resolves upon statin cessation or an autoimmune IMNM that may transform to an inflammatory myopathy. The different outcomes may be due to distinct immune phenotypes of the patients. One might contend that the pathological alterations in muscle were a persistence of acute statin-associated myonecrosis; however, this assertion is implausible since the clinical, $\mathrm{CK}$, and histological changes were seen long after statin cessation ( $89 \%$ had symptoms persisting even after the statin had been discontinued for $>5$ months). It would also not explain the flares that occurred when immunosuppressive therapies were tapered, nor the cases where the histology evolved toward greater inflammation (i.e., IMNM to PM transformation). Furthermore, attempts to taper off all immunosuppressive agents in all 26 patients led to clinical or biochemical (hyperCKemia) worsening. Collectively, the above data imply that statin exposure triggered a cascade of events leading to a perpetuating immunological disorder and not merely a circumscribed secondary inflammatory response to statin-induced myonecrosis. In this instance, other forms of toxic myopathies due to colchicine, amiodarone, ethanol, or highly active antiretrovirals, among many others, would be associated with dysimmune phenomena exaggerating the initial drug-induced myopathy, and they are not.

Two major concepts, which have supporting evidence from the literature, increase the plausibility of our hypothesis: (1) IMNM is caused by an immune-mediated mechanism just like PM, and (2) PM is a disease that can have various clinical and histopathological manifestations depending on time or location of biopsy. Three recent studies have described a total of $43\left(10,{ }^{55} 25,{ }^{27}\right.$ and $\left.8^{28}\right)$ patients who experienced persisting statin-associated IMNM. Researchers have reported associated inflammation in patients with IMNM, ${ }^{56,57}$ and another study showed five of 16 IMNM patients having endomysial and/or perivascular inflammatory cells. ${ }^{55}$ Moreover, 42 of the 43 patients in the previously mentioned studies improved both symptomatically and serologically after treatment with glucocorticoid and immunosuppressive medication (one patient did not receive immunosuppressive therapy) (Mammen, A., personal communication, 2011), and of the patients in the previously mentioned studies, 15 of 18 relapsed after tapering of immunosuppressive 
therapy. Finally, preliminary mechanistic evidence shows IMNM to be immune mediated. Mammen et al. (2011) showed that statins upregulate expression of HMGCR, the major target of new autoantibodies in patients with statin-associated IMNM, and that these autoantibodies may prolong immune response despite statin discontinuation by targeting the HMGCR, which becomes highly expressed on regenerating muscle fibres. ${ }^{32,58} \mathrm{We}$ recognize that antiHMGCR autoantibody testing would have aided in characterization of histopathologic phenotypes; however, this test was not performed and is a limitation of this study. However, anti-HMGCR autoantibodies have not yet been proven to be pathogenic: up to $33 \%$ of patients positive for the antibodies were statin naïve. Moreover, 38 of 53 patients had necrotizing changes and 10 of 53 had prominent perivascular and endomysial inflammation, supporting our hypothesis of changing histopathology. Finally, the autoantibodies cannot explain why some patients develop a self-limited toxicity, whereas others develop a persistent IMNM since they are not expressed until after the necrotic insult has occurred.

Finally, the notion that PM is a "patchy" disease is important to interpret variable manifestations presenting over time and anatomical locations. For example, in many instances, PM is mentioned as being a patchy disease, ${ }^{59}$ having patchy muscle involvement, ${ }^{60,61}$ or not having every abnormality presenting in every patient (i.e., clinical, histological, EMG, CK). ${ }^{62}$ Such variation may be one reason for our findings of histopathologic transition from one diagnosis to another, since five patients had subsequent biopsies in a different anatomical location, four of whom had changing diagnoses. In fact, in a comprehensive review, ${ }^{63}$ Dalakas states that misinterpretation of biopsies is the most common reason for misdiagnoses, especially those between sporadic IBM and PM, between PM or IMNM and inflammatory muscular dystrophies, and between PM and IMNM. This assertion supports the concept that the increasingly prevalent histopathologic diagnosis of IMNM is not necessarily an immutably distinct entity but rather a temporal (i.e., found intermittently within the time course of patients with PM) and/or spatial (i.e., "patchy") pathologic manifestation of an autoimmune myopathy spectrum.

This report links statins to the development of an autoimmune myopathy, with and without inflammatory findings, that persists or worsens even after statin withdrawal and responds to immunosuppressive therapy. Our data showing that poststatin myopathies can evolve into different histological diagnoses and yet still respond in a similar manner to the same disease-modifying agents increase the likelihood that statin-associated PM and IMNM are part of a single entity (i.e., can be lumped). Further studies, including the testing of anti-HMGCR autoantibodies in these patients, are needed. Because this test is now commercially available in Canada, it will further clarify this evolving area of neuromuscular medicine here. ${ }^{64}$

\section{ACKNOWLEDGEMENTS AND FUndING}

The authors thank Delores Reid, Linda Martin, and Anneke Nijenhuis for outstanding administrative assistance. Dr. Tarnopolsky holds a chair in Neuromuscular Disorders from McMaster Children's Hospital and Dr. Baker holds the Hamilton Hospital Assessment Center Chair in Neuromuscular Disorders. Partial funding came from "Jay's Drive Fore MD Gold Tournament".

\section{Disclosures}

The authors declare no conflicts of interest.

\section{REFERENCES}

1. Vaughan CJ, Gotto AM, Jr. Update on statins: 2003. Circulation. 2004;110:886-92.

2. Jackevicius CA, Cox JL, Carreon D, et al. Long-term trends in use of and expenditures for cardiovascular medications in Canada. CMAJ. 2009;181:E19-28.

3. Stagnitti MN. Trends in statins utilization and expenditures for the U.S. civilian noninstitutionalized population, 2000 and 2005. Statistical Brief \#205. Agency for Healthcare Research and Quality; 2008.

4. Josan K, Majumdar SR, McAlister FA. The efficacy and safety of intensive statin therapy: a meta-analysis of randomized trials. CMAJ. 2008;178:576-84.

5. Baker SK, Samjoo IA. A neuromuscular approach to statin-related myotoxicity. Can J Neurol Sci. 2009;35:8-21.

6. Omar MA, Wilson JP. FDA adverse event reports on statinassociated rhabdomyolysis. Ann Pharmacother. 2002;36:288-95.

7. Ucar M, Mjorndal T, Dahlqvist R. HMG-CoA reductase inhibitors and myotoxicity. Drug Saf. 2000;22:441-57.

8. Buettner C, Davis RB, Leveille SG, Mittleman MA, Mukamal KJ. Prevalence of musculoskeletal pain and statin use. J Gen Intern Med. 2008;23:1182-6.

9. Bruckert E, Hayem G, Dejager S, Yau C, Begaud B. Mild to moderate muscular symptoms with high-dosage statin therapy in hyperlipidemic patients - the PRIMO study. Cardiovasc Drugs Ther. 2005;19:403-14

10. Nichols GA, Koro CE. Does statin therapy initiation increase the risk for myopathy? An observational study of 32,225 diabetic and nondiabetic patients. Clin Ther. 2007;29:1761-70.

11. Phillips PS, Haas RH, Bannykh S, et al. Statin-associated myopathy with normal creatine kinase levels. Ann Intern Med. 2002;137:581-5.

12. Dobkin BH. Underappreciated statin-induced myopathic weakness causes disability. Neurorehabil Neural Repair. 2005;19:259-63.

13. Agostini JV, Tinetti ME, Han L, McAvay G, Foody JM, Concato J. Effects of statin use on muscle strength, cognition, and depressive symptoms in older adults. J Am Geriatr Soc. 2007;55:420-5.

14. Graham DJ, Staffa JA, Shatin D, et al. Incidence of hospitalized rhabdomyolysis in patients treated with lipid-lowering drugs. JAMA. 2004;292:2585-90.

15. Soininen K, Niemi M, Kilkki E, Strandberg T, Kivisto KT. Muscle symptoms associated with statins: a series of twenty patients. Basic Clin Pharmacol Toxicol. 2006;98:51-4.

16. Baker SK. Molecular clues into the pathogenesis of statin-mediated muscle toxicity. Muscle Nerve. 2005;31:572-80.

17. Fauchais AL, Iba Ba J, Maurage P, et al. Polymyositis induced or associated with lipid-lowering drugs: five cases. Rev Med Interne. 2004;25:294-8

18. Riesco-Eizaguirre G, Arpa-Gutierrez FJ, Gutierrez M, Toribio E. Severe polymyositis with simvastatin use. Rev Neurol. 2003;37:934-6

19. Giordano N, Senesi M, Mattii G, Battisti E, Villanova M, Gennari C. Polymyositis associated with simvastatin. Lancet. 1997;349: $1600-1$.

20. Takagi A, Shiio Y. Pravastatin-associated polymyositis, a case report. Rinsho Shinkeigaku. 2004;44:25-7.

21. Vasconcelos OM, Campbell WW. Dermatomyositis-like syndrome and HMG-CoA reductase inhibitor (statin) intake. Muscle Nerve. 2004;30:803-7.

22. Hill C, Zeitz C, Kirkham B. Dermatomyositis with lung involvement in a patient treated with simvastatin. Aust $\mathrm{N} \mathrm{Z} \mathrm{J} \mathrm{Med.}$ 1995;25:745-6.

23. Khattak FH, Morris IM, Branford WA. Simvastatin-associated dermatomyositis. Br J Rheumatol. 1994;33:199.

24. Noel B, Cerottini JP, Panizzon RG. Atorvastatin-induced dermatomyositis. Am J Med. 2001;110:670-1.

25. Rodriguez-Garcia JL, Serrano Commino M. Lovastatin-associated dermatomyositis. Postgrad Med J. 1996;72:694.

26. Zuech P, Pauwels C, Duthoit C, et al. Pravastatin-induced dermatomyositis. Rev Med Interne. 2005;26:897-902.

27. Grable-Esposito P, Katzberg HD, Greenberg SA, Srinivasan J, Katz J, Amato AA. Immune-mediated necrotizing myopathy associated with statins. Muscle Nerve. 2010;41:185-90. 
28. Needham M, Fabian V, Knezevic W, Panegyres P, Zilko P, Mastaglia FL. Progressive myopathy with up-regulation of MHC-I associated with statin therapy. Neuromuscul Disord. 2007;17: 194-200.

29. Dubowitz V, Sewry CA, Hohlfeld R, Dalakas MC. Muscle biopsy: a practical approach; structural and molecular basis of skeletal muscle disease; sporadic inclusion body myositis-diagnosis, pathogenesis and therapeutic strategies. Nat Clin Pract Neurol. 2006;21;221;437-9, 227;447.

30. Mammen AL. Dermatomyositis and polymyositis: clinical presentation, autoantibodies, and pathogenesis. Ann N Y Acad Sci. 2010;1184:134-53.

31. Weihl CC, Pestronk A. Sporadic inclusion body myositis: possible pathogenesis inferred from biomarkers. Curr Opin Neurol. 2010;23:482-8.

32. Mammen AL, Chung T, Christopher-Stine L, et al. Autoantibodies against 3-hydroxy-3-methylglutaryl-coenzyme a reductase in patients with statin-associated autoimmune myopathy. Arthritis Rheum. 2011;63:713-21.

33. Chatzizisis YS, Koskinas KC, Misirli G, Vaklavas C, Hatzitolios A, Giannoglou GD. Risk factors and drug interactions predisposing to statin-induced myopathy: implications for risk assessment, prevention and treatment. Drug Saf. 2010;33:171-87.

34. Golomb BA, Evans MA. Statin adverse effects: a review of the literature and evidence for a mitochondrial mechanism. Am J Cardiovasc Drugs. 2008;8:373-418.

35. Corsini A, Ceska R. Drug-drug interactions with statins: will pitavastatin overcome the statins' Achilles' heel? Curr Med Res Opin. 2011;27:1551-62.

36. Tarnopolsky MA, Pearce E, Smith K, Lach B. Suction-modified Bergstrom muscle biopsy technique: experience with 13,500 procedures. Muscle Nerve. 2011;43:717-25.

37. Dubowitz V, Sewry CA. Histological and histochemical stains and reactions. In: Dubowitz V, Sewry CA, editors. Muscle biopsy: A practical approach. 3rd ed. St. Louis:Saunders Elsevier; 2007. pp. 21-39.

38. Hoogendijk JE, Amato AA, Lecky BR, et al. 119th ENMC international workshop: trial design in adult idiopathic inflammatory myopathies, with the exception of inclusion body myositis, 10-12 October 2003, Naarden, The Netherlands. Neuromuscul Disord. 2004; $14: 337-45$

39. Bohan A, Peter JB. Polymyositis and dermatomyositis. N Engl J Med. 1975;292:344-7, 403-7.

40. Hohlfeld R. Polymyositis and dermatomyositis. In: Karpati G, editor. Structural and molecular basis of skeletal muscle diseases. Basel: ISN Neuropath Press; 2002. pp. 221-7.

41. Health, United States. 2010: with special feature on death and dying. Hyattsville, Md.: National Center for Health Statistics, 2011. p. 26.

42. Antons KA, Williams CD, Baker SK, Phillips PS. Clinical perspectives of statin-induced rhabdomyolysis. Am J Med. 2006;119: 400-409.

43. Schech S, Graham D, Staffa J, et al. Risk factors for statin-associated rhabdomyolysis. Pharmacoepidemiol Drug Saf. 2007;16:352-8.

44. Rider LG, Miller FW. Deciphering the clinical presentations, pathogenesis, and treatment of the idiopathic inflammatory myopathies. JAMA. 2011;305:183-90.
45. Limaye VS, Blumbergs P, Roberts-Thomson PJ. Idiopathic inflammatory myopathies. Intern Med J. 2009;39:179-90.

46. Cox S, Limaye V, Hill C, Blumbergs P, Roberts-Thomson P. Idiopathic inflammatory myopathies: diagnostic criteria, classification and epidemiological features. Int J Rheum Dis. 2010;13:117-24.

47. Chow SC. Immunomodulation by statins: mechanisms and potential impact on autoimmune diseases. Arch Immunol Ther Exp (Warsz). 2009;57:243-51.

48. Gurevich VS, Shovman O, Slutzky L, Meroni PL, Shoenfeld Y. Statins and autoimmune diseases. Autoimmun Rev. 2005;4:123-9.

49. Sailler L, Pereira C, Bagheri A, et al. Increased exposure to statins in patients developing chronic muscle diseases: a 2-year retrospective study. Ann Rheum Dis. 2008;67:614-9.

50. Arora M, Chen L, Paglia M, et al. Simvastatin promotes Th2-type responses through the induction of the chitinase family member Ym1 in dendritic cells. Proc Natl Acad Sci U S A. 2006;103:7777-82.

51. Dunn SE, Youssef S, Goldstein MJ, et al. Isoprenoids determine Th1/Th2 fate in pathogenic $\mathrm{T}$ cells, providing a mechanism of modulation of autoimmunity by atorvastatin. J Exp Med. 2006; 203:401-12.

52. Stupka N, Tarnopolsky MA, Yardley NJ, Phillips SM. Cellular adaptation to repeated eccentric exercise-induced muscle damage. J Appl Physiol. 2001;91:1669-78.

53. Neal RC, Ferdinand KC, Ycas J, Miller E. Relationship of ethnic origin, gender, and age to blood creatine kinase levels. Am J Med. 2009; $122: 73-8$.

54. Fung EC, Crook MA. Statin myopathy: a lipid clinic experience on the tolerability of statin rechallenge. Cardiovasc Ther. 2011;30: e212-8.

55. Christopher-Stine L, Casciola-Rosen LA, Hong G, Chung T, Corse AM, Mammen AL. A novel autoantibody recognizing $200-\mathrm{kd}$ and $100-\mathrm{kd}$ proteins is associated with an immune-mediated necrotizing myopathy. Arthritis Rheum. 2010;62:2757-66.

56. Mammen AL, Amato AA. Statin myopathy: a review of recent progress. Curr Opin Rheumatol. 2010;22:644-50.

57. Khan S, Christopher-Stine L. Polymyositis, dermatomyositis, and autoimmune necrotizing myopathy: clinical features. Rheum Dis Clin North Am. 2011;37:143-58, v.

58. Morikawa S, Murakami T, Yamazaki H, et al. Analysis of the global RNA expression profiles of skeletal muscle cells treated with statins. J Atheroscler Thromb. 2005;12:121-31.

59. Riddoch D, Morgan-Hughes JA. Prognosis in adult polymyositis. J Neurol Sci. 1975;26:71-80.

60. McKown KM. Chapter 86: polymyositis and dermatomyositis. In: Alguire PM, editor. Internal medicine essentials for clerkship students 2. Washington, D.C.:ACP Press; 2009. pp. 317-8.

61. Dalakas MC, Karpati G. Chapter 22: inflammatory myopathies. In: Karpati G, Hilton-Jones D, Bushby K, Griggs RC, editors. Disorders of voluntary muscle. 8th ed. Cambridge:Cambridge University Press; 2010. pp. 427-52.

62. DeVere R, Bradley WG. Polymyositis: its presentation, morbidity and mortality. Brain. 1975;98:637-66.

63. Dalakas MC. Review: an update on inflammatory and autoimmune myopathies. Neuropathol. Appl Neurobiol. 2011;37:226-42.

64. Mahler M, Pierangeli S, Meroni P, Fritzler MJ. Autoantibodies in Systemic Autoimmune Disorders. J Immunol Res. 2014;263-91. 